\title{
Benefícios e comparação na atuação do cateter nasal e da ventilação não invasiva em pediatria: uma revisão sistemática
}

\author{
Benefits and comparison in the performance of the nasal catheter and non-invasive \\ ventilation in pediatrics: a systematic review
}
Beneficios y comparación en el rendimiento del catéter nasal y la ventilación no invasiva en pediatría: una revisión sistemática

Silvia Ataides Alves Santana ${ }^{1 *}$, Ezucleide Carvalho Camada de Oliveira ${ }^{1}$, Marcos Cesar Ramos Mello $^{1}$, Dayane Cristina Pinto Neves ${ }^{1}$, Állef Diego Bonfim de Andrade ${ }^{2}$, Cícero Duarte da Cunha ${ }^{2}$, Maria Priscilla de Souza Pereira Albuquerque Carvalho², Nária Germana Basílio Ramalho de Alencar Paiva ${ }^{1}$, Luciana Gusmão Medeiros ${ }^{1}$, Rodrigo Crestoni Freire de Andrade ${ }^{2}$.

\section{RESUMO}

Objetivo: Verificar a eficácia e a atuação do Cateter Nasal de Alto Fluxo e da Ventilação Não Invasiva por pressão positiva nos pacientes pediátricos, visando uma opinião comparativa entre eles. Métodos: foram pesquisadas as bases de dados "online", Vapotherm, ScienceDirect, Medical Literature Analysis and Retrieval System Online (MEDLINE), Biblioteca Virtual em Saúde (BVS) e Scientific Electronic Library Online (SCIELO). Foi realizado um levantamento bibliográfico dos estudos relevantes publicados, por meio de palavras-chave sobre o tema, sendo selecionados artigos que preenchiam os seguintes critérios: estudos clínicos e terem sido publicado entre 2009 e 2019. Foram excluídas as duplicidades, os artigos sem resumo, as revisões de literatura e os sem acesso livre. Um total de 9 (nove) artigos foram selecionados atendendo a esses critérios. Resultados: Foram analizados nove pesquisas, que analisavam os benefícios do Cateter Nasal de Alto Fluxo (CNAF) e da Pressão Positiva Contínua nas Vias Aéreas (CPAP) nas patologias respiratórias. Consideraçãoes finais: Ambos os recursos são eficazes na prevenção da Intubação Orotraqueal e prevenção de internação de crianças em Unidades de Terapia Intensiva Pediátrica, não havendo superioridade entre elas.

Palavras-chave: Cateteres, Pressão Positiva Contínua nas Vias Aéreas, Pediatria, Insuflação.

\section{ABSTRACT}

Objective: To verify the effectiveness and performance of the High Flow Nasal Catheter and Non-Invasive Ventilation by positive pressure in pediatric patients, aiming at a comparative opinion between them. Methods: "online", Vapotherm, ScienceDirect, Medical Literature Analysis and Retrieval System Online (MEDLINE), Virtual Health Library (VHL) and Scientific Electronic Library Online (SCIELO) databases were searched. A bibliographic survey of the relevant published studies was carried out, using keywords on the topic, with articles that met the following criteria being selected: clinical studies and having been published between 2009 and 2019. Duplications, articles without abstract, literature reviews and those without free access. A total of 9 (nine) articles were selected according to these criteria. Results: Nine studies were analyzed, which analyzed the benefits of the High Flow Nasal Catheter (CNAF) and the Continuous Positive Airway Pressure (CPAP) in respiratory pathologies. Final considerations: Both resources are effective in preventing Oro-tracheal intubation and preventing hospitalization of children in Pediatric Intensive Care Units, with no superiority between them.

Key words: Catheters, Continuous Positive Airway Pressure, Pediatrics, Insufflation.

${ }^{1}$ Faculdade Inspirar, Porto Velho - RO. *E-mail: silvinhapopcpma@yahoo.com.br

2 Hospital Regional de Cacoal. Cacoal - RO. 


\section{RESUMEN}

Objetivo: verificar la efectividad y el rendimiento del catéter nasal de alto flujo y la ventilación no invasiva mediante presión positiva en pacientes pediátricos, con el objetivo de obtener una opinión comparativa entre ellos. Métodos: se realizaron búsquedas en las bases de datos "en línea", Vapotherm, ScienceDirect, Medical Literature Analysis and Retrieval System (MEDLINE), Virtual Health Library (VHL) y Scientific Electronic Library Online (SCIELO). Se realizó una encuesta bibliográfica de los estudios publicados relevantes, utilizando palabras clave sobre el tema, y se seleccionaron artículos que cumplían con los siguientes criterios: estudios clínicos y publicados entre 2009 y 2019. Duplicaciones, artículos sin resumen, revisiones de literatura y aquellos sin acceso libre. Se seleccionaron un total de 9 (nueve) artículos de acuerdo con estos criterios. Resultados: se analizaron nueve estudios, que analizaron los beneficios del catéter nasal de alto flujo (CNAF) y la presión positiva continua en las vías respiratorias (CPAP) en patologías respiratorias. Consideraciones finales: Ambos recursos son efectivos para prevenir la intubación orotraqueal y prevenir la hospitalización de niños en unidades de cuidados intensivos pediátricos, sin superioridad entre ellos.

Palabras clave: Catéteres, Presión Positiva Contínua en las Vías Respiratorias, Pediatría, Insuflación.

\section{INTRODUÇÃO}

As principais causas de doenças entre as crianças menores de cinco anos são as Insuficiências Respiratórias Aguda (IRA), isso se deve principalmente as características peculiares relacionadas à anatomia do sistema respiratório da criança, que se encontra em constante desenvolvimento. A IRA é um evento bastante frequente em pediatria e corresponde a $50 \%$ das internações em Unidade de Terapia Intensiva Pediátrica (UTIP), sendo uma das principais causas de morbidade e mortalidade nesta população (OLIVEIRA JBS, SOARES MES, 2013).

O tratamento estabelecido na insuficiência respiratória aguda associada as infecções de vias aéreas inferiores precisa ser precoce para restaurar a ventilação e a oxigenação tecidual. Muitas vezes a oxigenoterapia, hidratação e aspiração de secreções se fazem necessárias. Nos casos mais graves, tornase necessário medidas de suporte ventilatório, como a ventilação não invasiva (VNI) e a Ventilação Mecânica Invasiva (PIVA JP, et al., 1998).

Destaca-se como peculiaridades do sistema respiratório de lactentes e crianças menores, o menor diâmetro das vias aérea, que predispõe as obstruções; imaturidade da musculatura diafragmática e intercostal; canais de Lambert e Poros de Kohn pouco desenvolvidos, não ocorrendo a ventilação colateral; incoordenação tóraco-abdominal durante o sono REM (Rapid Eye Movement); caixa torácica mais complacente; pulmões menos elástico com complacência pulmonar diminuída;maior vulnerabilidade às infecções, por imaturidade do sistema imunológico ainda em desenvolvimento. Devido as essas peculiaridades as crianças são mais susceptíveis a apresentar obstrução das vias aéreas, fadiga muscular, dificuldade de higiene brônquica e formação de atelectasias (INAFUKO EM, 2019 apud PIVA JP, et al., 1997).

As principais causas de internação de crianças em UTI, segundo Batista NOW, et al. (2015) foram sepse, pneumonia, bronquiolite, asma e cardiopatias congênitas. Destacando as doenças respiratórias, em segundo, terceiro e quarto lugar. O suporte ventilatório não invasivo pode ser usado em lactentes e crianças com condições agudas e crônicas que decorrentes de IRA. Recurso muito utilizado previamente com muitas evidências em pacientes neonatais e adultos. Recentemente as pesquisas destacam o importante papel desse tipo de suporte também em pacientes pediátricos (FEDOR KL, 2017).

Os sistemas de Cânula Nasal de Alto Fluxo (CNAF) vêm sendo muito utilizado atualmente em pacientes críticos de todas as idades, desde recém-nascidos até o público adulto. Pode ser utilizado em todo tipo de UTI, tais como: neonatal, pediátrica, médica e cirúrgica, intermediária e salas de emergência (SLAIN KN, et al., 2017). A oxigenoterapia nasal de alto fluxo (ONAF) através da CNAF fornece oxigênio aquecido e umidificado com o objetivo melhorar a oxigenação e eliminar o dióxido de carbono (CO2) reduzindo o 
espaço morto, o que leva a atenuação frequência respiratória $(F R)$ e esforço respiratório, gerar pressão positiva expiratória final (PEEP) e proporcionar maior conforto respiratório (DRES M, DEMOULE A, 2017).

Evidências científicas mostram que os efeitos benéficos do CNAF no organismo se dá possivelmente devido aos mecanismos que levam a uma resistência inspiratória reduzida, a eliminação do espaço morto anatômico nasofaríngeo, um trabalho metabólico relacionado ao condicionamento de gás reduzido (disponibilidade de gás totalmente condicionado para as vias aéreas, reduzindo as perdas insensíveis de água e o custo de energia para o aquecimento do gás inspirado à temperatura do corpo), melhor condutância das vias aéreas e do transporte mucociliar e fornecimento de baixos níveis de pressão positiva nas vias aéreas (SLAIN KN, et al., 2017).

Trata-se de uma técnica recente e inovadora, mais simples de usar e aplicar do que VNI, sendo uma alternativa útil e eficaz para a IRA por ser melhor tolerada (evitando claustrofobia), fornecendo alta fração de oxigênio inspirado ( $\mathrm{FiO} 2$ ), gerando baixo nível de pressão positiva e melhorando propriedades mecânicas pulmonares (BONFIM ES, 2018). A fonte pressurizada de oxigênio e ar, o reservatório de água esterilizada conectada a um aquecedor e umidificador, o circuito isolado e/ou aquecido e a cânula não oclusiva fazem parte da composição do Sistema de CNAF que fariam de acordo com o fabricante (SLAIN KN, et al., 2017).

Para o uso da CNAF o paciente necessita estar respirando espontaneamente, alerta e consciente com vias aéreas livres hemodinamicamente estável e é utilizado quando paciente apresentar sinais e sintomas tais como: dispneia, intolerância a VNI, hipercapnia, hipoxemia refratária, aumento do trabalho cardíaco e aumento do trabalho respiratório. Dentre as patologias indicadas para o uso desse dispositivo incluem a pneumonia, bronquite, bronquiolite, exacerbação aguda de Doença Pulmonar Obstrutiva Crônica (DPOC), Insuficiência Cardíaca Congestiva (ICC) leve ou moderada, asma, pré-transplante cardíaco, pacientes com desmames difícil da Ventilação Mecânica, IRA, pacientes em Cuidados Paliativos (CP) e os Cirúrgicos pós extubação. Estando contra-indicado para paciente com IRA grave, lesão de face que impeça a adaptação da terapia, cirurgias que faça tamponamento nasal, instabilidade hemodinâmica, rebaixamento do nível de consciência e impossibilidade de proteção de vias respiratórias com risco de broncoaspirações (SARMENTO GJV, et al., 2020).

O sucesso do uso do CNAF pode ser avaliado com base na frequência cardíaca (FC) e na FR na primeira hora e os que respondem bem ao tratamento podem receber cuidados com segurança nas enfermarias pediátricas, não sendo necessária internação em UTIP, o que resulta na redução de custos com cuidados intensivos (MANLEY BJ, et al., 2013). Outro recurso amplamente utilizado nas IRA é a VNI, que promove a entrada de ar nos pulmões por meio de uma máquina que gera pressão positiva, através de uma interface. Uma alternativa ao uso da VMI que além de ser um procedimento invasivo, está associado a complicações graves e que podem comprometer a evolução clínica do paciente. A maior vantagem dessa ventilação é não demandar de dispositivo invasivo para ventilar o paciente. As interfaces são dispositivos colocados no final do circuito do ventilador em contato com o paciente, podem ser de diferentes tipos, tais como prong, capacete e máscaras nasal, facial, oro-nasal e facial total. A indicação da interface depende do conforto do paciente, da redução do espaço morto e do impedimento de vazamentos (RAHAL L, et al., 2005).

Uma das modalidades de VNI mais utilizada e que marcou a entrada do fisioterapeuta na UTI é a ventilação por Pressão Positiva Contínua nas Vias Aéreas (CPAP). Segundo as Recomendações Brasileiras de Ventilação Mecânica (2014), é necessário para uso do CPAP a escolher interface adequada que possa se adaptar bem à face do paciente, a utilizar interfaces sem compressão nasal se o tempo estimado de VNI for >24 a 48 horas, usar interface com válvula de PEEP se a opção for CPAP com gerador de fluxo e utilizar máscara conectada a circuito duplo quando for ventilador mecânico de UTI (microprocessado convencional) e máscara para circuito único quando ventilador específico (CPAP) (BARBAS CS, et al., 2014).

Possuem grau de recomendação "A" para o uso de CPAP, as patologias: Doença Pulmonar Obstrutiva Crônica (DPOC), Edema Pulmonar Cardiogênico (EAPC), Insuficiência Respiratória Pós-extubação. Sendo contraindicações para seu uso: distensão abdominal ou vômito pelo risco de aspiração, trauma de face, lesão aguda e/ou sangramento de via aérea. Outras contraindicações ao uso do CPAP são: diminuição do 
nível de consciência, sonolência, agitação, confusão ou recusa do paciente, instabilidade hemodinâmica, arritmias, obstruções de via aérea superior, tosse ineficaz, sangramento digestivo alto, infarto agudo do miocárdio, pós-operatório de face, via aérea superior ou esôfago. O uso é controverso nos casos de cirurgia gástrica e gravidez (SCHETTINO GPP, et al., 2007).

O presente artigo se justifica pela necessidade de pesquisar mais sobre os benefícios do Cateter Nasal de Alto-fluxo e da Ventilação Não-Invasiva por Pressão Positiva e comparar a eficácia entre eles no tratamento de doenças com sintomas respiratórios na população pediátrica, com ênfase no atendimento a pacientes internados na UTI pois, trata-se de recurso utilizado na prevenção das IOT e de complicações a elas relacionadas, sendo assim, tem como objetivo revisar na literatura acerca do tema envolvendo estudos dos últimos 10 anos.

\section{MÉTODOS}

Trata-se de uma Revisão Sistemática da Literatura, por meio da busca de informações relevantes ao tema da pesquisa nas bases de dados foram pesquisadas as bases de dados "online", Vapotherm, ScienceDirect, Medical Literature Analysis and Retrieval System Online (MEDLINE), Biblioteca Virtual em Saúde (BVS) e Scientific Electronic Library Online (SCIELO). Para a construção da pergunta de pesquisa e da busca bibliográfica de evidência utilizou-se a estratégia PICO representada por quatro componentes: Paciente, Intervenção, Comparação e "Outcomes" (desfecho) (SANTOSCMC; PIMENTA CAM; NOBRE MRC, 2007). Para delimitar o objeto dessa pesquisa optou-se por selecionar estudos clínicos na forma de artigos publicados em periódicos nacionais e internacionais, tendo considerado o ano de 2009 como 0 período de início para tal levantamento. Esta opção se faz devido ao fato de ter acesso aos estudos dos últimos 10 anos.

Foram incluídos os estudos clínicos de caso, controlados ou randomizados em crianças com idade entre 29 dias e 18 anos, realizados nos últimos 10 (dez) anos, que utilizaram a VNI e/ou CNAF no tratamento de sintomas respiratórios, por meio das palavras-chave. Foram excluídas as duplicidades, os artigos sem resumo, as revisões de literatura e os sem acesso livre, os que envolviam Recém-Nascidos (RN) e adultos, e, as publicações anteriores a 10 (dez) anos (Figura 1).

Figura 1 - Fluxograma representando os critérios de inclusão e de exclusão.

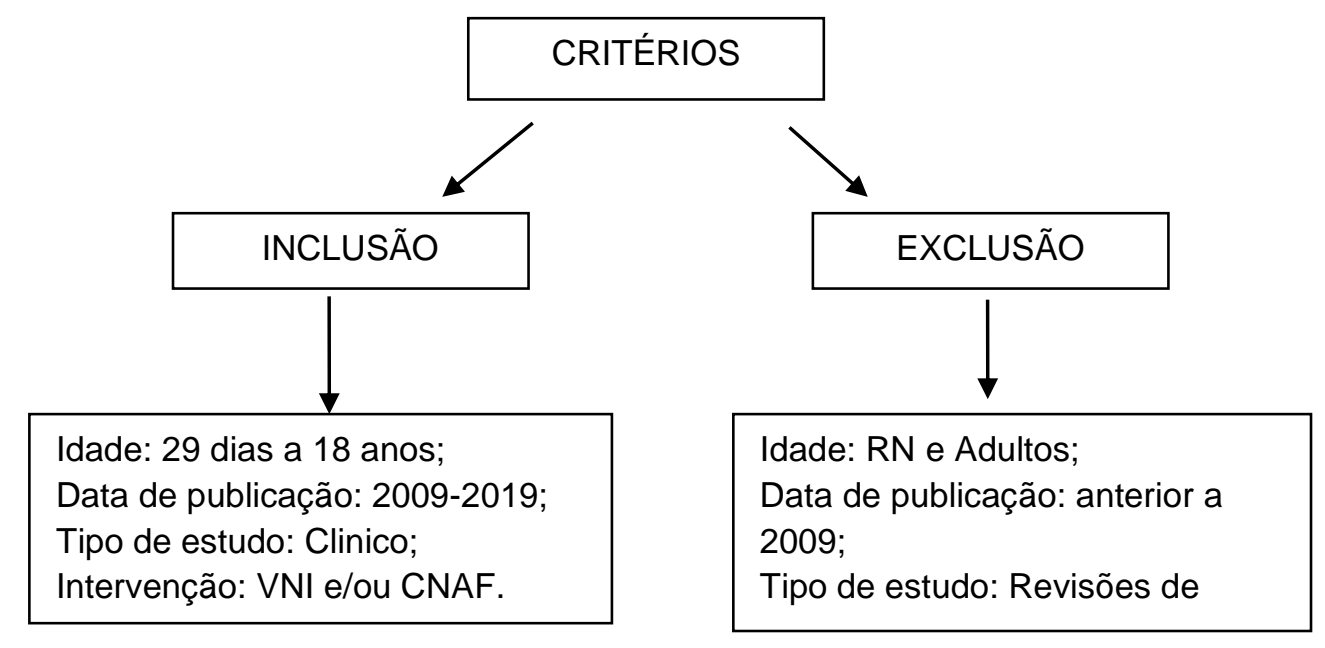

Fonte: Santana SAA, et al., 2020.

As palavras-chave utilizadas para a busca de artigos sobre "o uso do cateter nasal de alto-fluxo e a ventilação não-invasiva por pressão positiva em pediatria" pertencem a BSV e foram: Cateteres, Pressão Positiva Contínua nas Vias Aéreas, Pediatria e Insuflação, utilizando a interseção dos conjuntos (descritores do assunto, tipo de publicação e ano de publicação). 


\section{RESULTADOS}

$\mathrm{Na}$ combinação dos descritores "Cateter Nasal de Alto-fluxo, Ventilação Não-invasiva por Pressão Positiva, Estudos clínicos e Pediatria", foram encontradas dois na PubMed, dois na SciELO, um na Lilacs, dois na ScienceDirect, um na Vapotherm (estudos de caso) e um Medicina Infantil. Após a exclusão dos artigos não apresentados na íntegra, e cuja temática não contemplava a abordagem desse estudo, permaneceram nove artigos, conforme (Quadro 1). Os estudos selecionados foram realizados em diferentes países, sendo dois nos Estados Unidos, dois Uruguai, um Argentina, dois Espanha e dois no Brasil. Com relação ao ano de publicação, esses se encontraram na faixa pré-estabelecida pelos critérios do estudo entre 2009 a 2019. Quando comparado o uso do Cateter Nasal de Alto-fluxo com a Ventilação Não-invasiva por Pressão Positiva, apenas um artigo, mostrou que ambos apresentaram melhora dos sintomas. Nos estudos que utilizaram técnica isolada de CNAF ou VNI, não houve diferença significativa nos benefícios oferecidos por elas, não existindo superioridade entre elas.

Quadro 1 - Descrição dos artigos de pesquisa selecionados.

\begin{tabular}{|c|c|c|c|c|}
\hline Autor e ano & Tipo de estudo & Amostra & $\begin{array}{l}\text { Intervenção } \\
\text { / Patologias }\end{array}$ & Resultados \\
\hline $\begin{array}{l}\text { Morosini F, } \\
\text { et al. (2018) }\end{array}$ & $\begin{array}{l}\text { Retrospectivo, } \\
\text { descritivo }\end{array}$ & 650 crianças & CNAF/CPAP & $\begin{array}{ll}\text { - } & \text { Redução da necessidade de VMI e } \\
\text { internação na UTI }\end{array}$ \\
\hline $\begin{array}{l}\text { Ureta BL, et } \\
\text { al. (2011) }\end{array}$ & $\begin{array}{l}\text { Observacional } \\
\text { analítico, } \\
\text { retrospectivo }\end{array}$ & 340 crianças & CPAP & $\begin{array}{l}\text { - } \text { Fatores de risco de falha de CPAP: } \\
\text { atelectasia, idade menor ou igual } \\
\text { seis meses, comorbidades } \\
\text { infecciosas, disfunção ventricular e } \\
\text { obstrução de vias aéreas } \\
\text { superiores (VAS) }\end{array}$ \\
\hline $\begin{array}{l}\text { Junior JC, } \\
\text { et al. (2018) }\end{array}$ & Relato de caso & $\begin{array}{l}1 \text { lactente de } \\
8 \text { meses }\end{array}$ & $\begin{array}{l}\text { CNAF } \\
\text { Bronquiolite }\end{array}$ & $\begin{array}{l}\text { - Redução a FC e FR } \\
\text { - Melhora do padrão respiratório } \\
\text { - Evitar a necessidade de re- } \\
\text { intubação }\end{array}$ \\
\hline $\begin{array}{l}\text { Martinez } \\
\text { GF, et al. } \\
\text { (2013) }\end{array}$ & Observacional & $\begin{array}{l}25 \text { crianças } \\
\text { menores de } \\
18 \text { meses. }\end{array}$ & $\begin{array}{l}\text { CNAF } \\
\text { Bronquiolite }\end{array}$ & $\begin{array}{l}\text { - } \quad \text { Melhor FC e FR; } \\
\text { - Menor necessidade de internações } \\
\text { em UTIP. }\end{array}$ \\
\hline $\begin{array}{l}\text { Tejera J, et } \\
\text { al. (2013) }\end{array}$ & Observacional & $\begin{array}{l}23 \text { crianças } \\
\text { menores de } \\
2 \text { anos. }\end{array}$ & $\begin{array}{l}\text { CNAF } \\
\text { Bronquiolite }\end{array}$ & $\begin{array}{l}\text { - Melhora de parâmetros clínicos; } \\
\text { - Redução da necessidade de } \\
\text { admissão em UTIP. }\end{array}$ \\
\hline $\begin{array}{l}\text { McGinley B, } \\
\text { et al. (2009) }\end{array}$ & Experimental & $\begin{array}{l}12 \text { crianças } \\
\text { com idade } \\
\text { média de } 10 \\
\text { anos. }\end{array}$ & $\begin{array}{l}\text { CNAF } \\
\text { Síndrome da } \\
\text { Apnéia } \\
\text { Obstrutiva } \\
\text { (ASO) } \\
\end{array}$ & $\begin{array}{l}\text { - Redução do trabalho respiratório; } \\
\text { - } \text { Aumento das reservas de oxigênio; } \\
\text { - Menor ocorrência de apnéia } \\
\text { obstrutiva. }\end{array}$ \\
\hline $\begin{array}{l}\text { Figueruelo } \\
\text { AG, et al. } \\
\text { (2011) }\end{array}$ & Observacional & 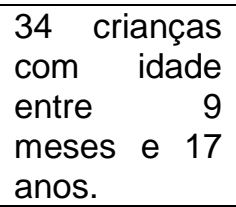 & $\begin{array}{l}\text { CNAF } \\
\text { IRA }\end{array}$ & $\begin{array}{l}\text { - Melhora clínica; } \\
\text { - Tolerância a retirada da Ventilação } \\
\text { Mecânica Invasiva (VMI). }\end{array}$ \\
\hline $\begin{array}{l}\text { Bressan S, } \\
(2013)\end{array}$ & $\begin{array}{l}\text { Observacional } \\
\text { prospectivo }\end{array}$ & 27 lactentes & $\begin{array}{l}\text { CNAF } \\
\text { Bronquiolite } \\
\text { moderada- } \\
\text { grave }\end{array}$ & $\begin{array}{l}\text { - } \begin{array}{l}\text { Melhora dos níveis de saturação } \\
\text { de oxigênio (SaO2); }\end{array} \\
\text { - } \begin{array}{l}\text { Diminuição tanto no } \\
\text { expirado; }\end{array} \\
\text { - } \begin{array}{l}\text { Redução } \\
\text { respiratória. da }\end{array} \\
\text { resequência }\end{array}$ \\
\hline $\begin{array}{l}\text { Perusso D, } \\
\text { et al. (2019) }\end{array}$ & Estudo de caso & $\begin{array}{l}1 \text { criança de } \\
10 \text { meses }\end{array}$ & $\begin{array}{l}\text { CNAF } \\
\text { (Vapotherm) } \\
\text { IRA }\end{array}$ & $\begin{array}{ll}\text { - } & \text { Melhora do desconforto } \\
& \text { respiratório em } 10 \text { minutos; } \\
\text { - } & \text { Redução da frequência } \\
\text { respiratória; Melhora da dispnéia e } \\
\text { da SaO2. }\end{array}$ \\
\hline
\end{tabular}

Fonte: Santana SAA, et al., 2020. 


\section{DISCUSSÃO}

Em estudo observacional realizado por Martinez GF, et al. (2013) em um hospital terciário, foram incluídas 25 crianças menores que dezoito meses com diagnóstico de bronquiolite que necessitavam de oxigenoterapia ao tratamento com CNAF. Observaram que as crianças apresentaram melhora da frequência respiratória e cardíaca, reduzindo a necessidade de internação em UTIP.

Corroborando com estudo de Tejera J, et al. (2013) que observou os parâmetros clínicos e cardiológicos de 23 crianças menores de 2 anos com bronquiolites em tratamento com CNAF. Foi observado melhora significativa dos pacientes, onde apenas $22 \%$ evoluíram com necessidade de internação em UTIP.

Estudo de Júnior JC, et al. (2017) analisou os resultados da implantação do CNAF em uma Unidade de Pronto Atendimento (UPA) de Morumbi, que previamente utilizava do CPAP para tratamento de IRA para diminuir seus sintomas e evitar a IOT. Observaram que a IOT foi reduzida em $100 \%$ quando aplicado um protocolo assistencial multiprofissional, com equipe treinada e capacitada para usar o CNAF, os pacientes apresentaram redução de $90 \%$ dos sintomas como uso de musculatura acessória, redução da FR e FC nos primeiros 10 minutos de uso, evoluindo para reversão total dos sinais clínicos em até 1 hora. A taxa de internação em UTIP reduziu $10 \%$ quando comparado com o período anterior a implantação do novo dispositivo.

Estudo experimental de McGinley B, et al. (2009) com 12 crianças com média de idade de 10 anos com síndrome da apnéia obstrutiva (ASO) em substituição ao CPAP (também indicado) devido a baixa adesão da técnica. Foi oferecido às crianças um fluxo de ar aquecido de 201/minuto por CNAF. Observaram que ocorreu redução da limitação do fluxo inspiratório, diminuição da FR e do trabalho inspiratório. Melhorou as reservas de oxigênio, diminuiu a excitação e também a ocorrência de apnéia obstrutiva.

Os benefícios do CNAF em crianças entre 9 meses e 17 anos foi analisado por Figueruelo AG, et al. (2011) em um estudo clínico prospectivo observacional, no qual foram estudados 34 pacientes. As variáveis estudadas incluíram: idade, sexo, FR, FC, saturação de oxigênio (SaO2), gasometria, melhora clínica, tolerância, aparência de complicações e falha do tratamento. Observaram melhora clínica e/ou tolerância a retirada da $\mathrm{VMI}$ em aproximadamente $82 \%$ dos pacientes.

Em estudo de caso de Perusso D, et al (2019), uma lactente de 10 meses com sinais de IRA admitida em um serviço de emergência de um hospital de São Paulo, com suposta indicação de IOT, foi instituído o CNAF da marca Vapotherm aquecido e com fluxo entre 10 e 20 litros/minuto (baseado no peso da criança) aumentado gradativamente conforme tolerância e acomodação. Observou-se após 10 minutos de uso melhora do desconforto respiratório, redução da freqüência respiratória e cárdica e, aumento da SaO2.

Um estudo observacional prospectivo de Bressan S (2013) foi realizado em um centro acadêmico de cuidados terciários pediátricos na Itália no período de 2011 a 2012 em 27 lactentes (idade média de 1,3 meses) hospitalizados em enfermaria pediátrica com bronquiolite moderada- grave com objetivo de avaliar a efetividade do uso da CNAF nesta população bem como avaliar as alterações nos parâmetros ventilatórios antes e após o início do suporte ao CNAF. Foi observado que o uso da CNAF obteve melhora dos níveis de $\mathrm{SaO} 2$ e parece estar associada a uma diminuição tanto no $\mathrm{CO} 2$ quanto na FR.

Resultados semelhantes foram encontrados no estudo retrospectivo e descritivo de Morosini F (2018) em 650 crianças menores de 2 anos com infecções respiratórias baixas (IRAB) assistidos na Unidade Especial de Cuidados Respiratórios Agudo do Hospital de Referência Nacional de Pediatria no Uruguai onde receberam VNI (302 crianças) e CNAP (348 crianças) entre 2013 e 2016 sendo a maioria do sexo masculino (63\%) com bronquiolite (63\%) e 60\% com Vírus Respiratório Sincicial (VRS), 3 dias de duração ambas as técnicas, a média de idade das crianças que receberam VNI em torno de 12 meses e CNAF 11 meses. Com o uso dessas técnicas para cuidados moderados em crianças com IRAB foi observado diminuição da necessidade de VMI e internação na UTI.

Estudo observacional, analítico e retrospectivo de Ureta BL (2011) em 340 crianças (182 meninas e 158 meninos) com idade média 4,2 anos internadas na UTI 35 do Hospital Garrahan (2005-2008), com indicação de VNI por insuficiência cardíaca (IC) como resgate pós extubação e desmame no pré-operatório e pós-operatório com objetivo de pesquisar os fatores de risco que levam a falha de VNI. Nesse estudo os 
fatores de risco observados foram os seguintes: atelectasia, idade menor ou igual seis meses, comorbidades infecciosas, disfunção ventricular e obstrução de vias aéreas superiores (VAS).

De acordo com Slain KN, et al. (2017) cada vez mais evidências mostram que o CNAF exerce possíveis efeitos benéficos na redução da resistência inspiratória, elimina o espaço morto anatômico nasofaringe, reduz o gasto energético, melhora a condutância das vias aéreas e o transporte mucociliar, fornece baixas pressões positiva nas vias aéreas. Diferente do CPAP o CNAF não permite regular ou determinar a quantidade de pressões positivas nas vias aéreas.

Uma desvantagem ao uso do CNAF é o alto custo e curta durabilidade do circuito, possuindo como vantagem aos demais dispositivos de oxigenoterapia o fornecimento de fluxos elevados (de até 50 a 60 L/min) e fração inspirada de oxigênio de até $100 \%$ de forma não invasiva. A VNI em contrapartida é um recurso barato, eficaz e de simples aplicação no tratamento de IRA em pacientes que não apresentam descompensação hemodinâmica, tendo como desvantagens a distensão gástrica, hipoxemia transitória e necrose de pele facial devido a adaptação da interface (YUSTE ME, et al., 2019; SILVA DCB, et al., 2003).

Segundo Bonfim ES (2018) quando comparado a VNI com o CNAF, esta é melhor tolerada, mais simples de usar e aplicar, sendo uma alternativa confiável para a IRA. A VNI sempre foi a primeira escolha de suporte respiratório para manter ventilação e oxigenação adequada, porém se torna inviável quando não é tolerada pelo paciente. Em contrapartida a utilização do CNAF tornou-se mais rotineira devido a sua maior aceitabilidade, além de proporcionar igual vantagens na melhora clínica do paciente.

De acordo com Pires $\mathrm{P}$, et al. (2018) o uso do CNAF representa um avanço dentre as possibilidades de fornecimento de suporte ventilatório não invasivo do tratamento da IRA por apresentar uma melhora do conforto com baixos efeitos adversos. A técnica consiste em fornecer fluxo contínuo nas vias aéreas, iniciando com baixos fluxos e aumentando progressivamente permitindo uma maior adaptação do paciente ao dispositivo. O ar ofertado é aquecido podendo variar entre 21 e $100 \%$ de $\mathrm{FiO}$, sendo que o desmame deve iniciar-se a partir da redução da FiO2 e posteriormente do fluxo.

A VNI trata-se de um recurso ventilatório usado há mais de 30 anos nas diferentes patologias respiratórias que levam a IRA buscando evitar os efeitos deletérios causados pela VMI, proporcionando melhor conforto e reduzindo o tempo de permanência na UTI e custos com a internação. O sucesso da VNI depende dos critérios da indicação e do tipo de interface utilizada, sendo necessária uma boa acoplagem entre o paciente e o respirador. Deve-se atentar aos sinais clínicos de melhora ou piora do quadro para impedir falhas e minimizar os riscos de mortalidade (SANTOS LA, et al., 2017).

De acordo com Bocchile RLR (2018) o uso do CNAF não se mostrou eficaz na redução da necessidade de intubação ou reentubação de pacientes graves. Em contrapartida Rolim DS, et al. (2018) observou que o uso da VNI no pós-operatório de cirurgia cardíaca em crianças com IRA pós extubação obteve $61,8 \%$ de sucesso, não precisando serem reintubados, sendo considerada uma técnica eficaz para esta modalidade não apresentando eventos adversos que impossibilitassem a sua utilização.

\section{CONSIDERAÇÕES FINAIS}

Com base nas pesquisas encontradas, os resultados clínicos proporcionados pelo uso do CNAF e/ou da VNI são similares. O que difere um do outro, é a melhor aceitação do paciente (menor claustrofobia), o beneficio de oferecer ar aquecido (Vapotherm) e tempo de melhora dos sintomas, quando utilizado o CNAF. No que diz respeito a prevenção de IOT e necessidade de internação em UTI e melhora dos sintomas, não houve diferenças significativas entre o CPAP e o CNAF. Estudos que comparam o beneficio do CPAP e do CNAF são escassos, a maioria utilizou apenas uma técnica em sua pesquisa, sendo recomendado mais pesquisas que envolvam esse tema.

\section{REFERÊNCIAS}

1. BARBAS CS, et al. Recomendações brasileiras de ventilação mecânica 2013. Parte I. Revista Brasileira de Terapia Intensiva. 2014; 26(2):89-121.

REAS/EJCH | Vol.Sup.n.43 | e2977 | DOI: https://doi.org/10.25248/reas.e2977.2020 Página 7 de 9 
2. BATISTA NOW, et al. Perfil Clínico Epidemiológico de Pacientes Internados em Unidades de Cuidados Intensivos Pediátricos. Jornal of Human Growth and Development. 2015; 25(2): 187-193.

3. BENGUIGUI Y. As infecções respiratórias agudas na infância como problema de saúde pública. Bol. Pneumol. Sanit. v.10 n.1 Rio de Janeiro jun. 2002.

4. BOCCHILE RLR, et al. Efeitos do uso de cateter nasal de alto fluxo na intubação e na reintubação de pacientes críticos: revisão sistemática, metanálise e análise de sequência de ensaios. Revista Brasileira de Terapia Intensiva. Vol.30,no.4, São Paulo, Oct.-Dec. 2018.

5. BONFIM ES. A eficácia da cânula de alto fluxo alternativamente à ventilação não invasiva em pacientes hipoxêmicos. Revista Saúde e Meio Ambiente - RESMA, Três Lagoas, v. 6, n.1, pp. 56-70, janeiro julho. 2018. ISSN: 2447-8822.

6. BRESSAN $S$, et al. High-flow nasal cannula oxygen for bronchiolitis in a pediatric ward: a pilot study. Eur J Pediatr. 2013; 172 (12): 1649 - 1656.

7. DRES M; DEMOULE A. O que todo intensivista deve saber sobre oxigenoterapia nasal de alto fluxo em pacientes críticos. RevBras Ter Intensiva. 2017;29(4):399-403.

8. FEDOR KL. Noninvasive Respiratory Support in Infants and Children. Respir Care, v.62, n. 6, p.699-717, 2017.

9. FIGUERUELO AG, et al. Utilización de cánulas nasales de alto flujo para la ventilación no invasiva en niños. In: Anales de Pediatría [Internet]. Elsevier; 2011 [acesso em 2016, julho 26. P. 182-187.

10. GÓMEZ ES, et al. O uso de alto fluxo de oxigênio reduz a necessidade de intubação em comparação com o uso de ventilação mecânica não invasiva no paciente com sira leve? Med. crít. (Col. Mex. Med. Crít.) vol.30, no.5 Ciudad de México nov./dic. 2016.

11. INAFUKO, EM. Insuficência Respiratória Aguda na Infância. Departamento Nacional de Pós Graduação e Atualização. São Paulo, 2012.

12. JUNIOR JC, et al; Cânula nasal de alto fluxo pós- extubação traqueal em criança com obstrução das vias aéreas superiores: Relato de caso. Rev Paul Pediatr. 2018;36(3):372-375.

13. MANLEY BJet al.High-flow nasal cannulae in very preterm infants after extubation. N Engl J Med, 2013; 369(15): 1425- 1433.

14. MARTÍNEZ GF; SÁNCHEZ GMI; FERNÁNDEZ RR. Impacto clínico de la implantación de la ventilación por alto flujo de oxígeno en el tratamiento de la bronquiolitis en una planta de hospitalización pediátrica. An Pediatría. 2013;78(4):210-5.

15. MCGINLEY B, et al. Effect of a high-flow open nasal cannula system on obstructive sleep apnea in children. Pediatrics. 2009;124(1):179-88.

16. MELLO MCR, et al. Utilização da terapia de HVNI para reversão de pneumotórax: relato de caso.

17. MOROSINI Fet al. Ventilação não invasiva e oxigenoterapia de alto fluxo em crianças em consultórios de atenção de nível moderado. Experiência na Unidade de Cuidados Respiratórios Especiais Agudos do Hospital Pediátrico do CHPR durante 2013-2016. Arch. Pediatr. Urug.2018: 89 (2).

18. OLIVEIRA JBS; SOARES MES. Perfil epidemiológico da insuficiência respiratória aguda em crianças internadas na unidade de terapia intensiva de um hospital público da Paraíba. InterScientia, v. 1, n. 3, 2013. Acesso em: 25 de Outubro de 2019.

19. PERUSSO D, et al. Uso de Insuflação Nasal de Alta Velocidade (HVNI) na Reversão da Insuficiência Respiratória Aguda Multifatorial e Prevenção da Intubação Traqueal em um Paciente Pediátrico do Departamento de Emergência. Patient Case Studies. Mar 19.

20. PIRES P, et al. cannulas nasais de alto fluxo: Uma Alternative de Oxigenoterapia na Insuficiência Respiratória Aguda. Medicina Interna, Vol.25, no.2, ABR/JUN, 2018.

21. PIVA JP, et al. Síndrome do desconforto respiratório agudo (SDRA / SARA). In: Piva JP, Carvalho PRA, Garcia PCR. Terapia intensiva pediátrica, 4ª ed. Rio de Janeiro: MEDSI; 1997.

22. PIVA JP, et al. Insufciencia Respiratória na criança. Jornal de Pediatria, Vol. 74, Supl. 1, S99, 1998.

23. RAHAL L, et al. Ventilação não-invasiva: quando utilizar? Revista da Associação Médica Brasileira. Vol 51, n 5, São Paulo, Sept./Oct. 2005.

24. ROLIM DS, et al. A ventilação não invasiva como uma opção de suporte ventilação para pacientes pediátricos em pós operatório de cirurgia cardíaca com insuficiência respiratória. Universidade de São Paulo, 2018.

25. SAMPAIO RF; MANCINI MC. Estudos de revisão sistemática: um guia para síntese criteriosa da evidência científica. v. 11 n. 1, 2007 ISSN 1413-3555 Rev. bras. fisioter, São Carlos, v. 11, n. 1, p. 83-89, jan./fev. 2007.

26. SANTOS CMC, PIMENTA CAM, NOBRE MRC. A estratégia PICO para a construção da pergunta de pesquisa e busca de evidências. Rev Latino-am Enfermagem 2007 maio-junho; 15(3).

27. SANTOS LA, et al. Efeitos da ventilação não invasiva em pacientes com insuficiência respiratória aguda: uma revisão integrativa. ReonFacema. 2017, Jul- Set; 3(3): 642-648.

28. SARMENTO GJV, et al.. Ed 1. Editora Manole, 2020.

29. SCHETTINO GPP, et al. Ventilação mecânica não invasiva com pressão positiva. J. bras. pneumol. vol.33 suppl.2 São Paulo July 2007. 
30. SILVA DCB, et al. Ventilação não invasiva em pediatria. Jornal de Pediatria 79, S161-S168, 2003.

31. SINHA IP, et al. CPAP and High-Flow Nasal Cannula Oxygen in Bronchiolitis. Contemporary review sin critical care medicine [1 48 \# 3 c h e s t s September, 2015.

32. SLAIN KN; SHEIN SL; ROTTA AT. Uso de cânula nasal de alto fluxo no departamento de emergência pediátrica. J. Pediatr. (Rio J.) vol.93 supl.1 Porto Alegre, 2017.

33. TEJERA J, et al. Aplicación de oxigenoterapia de alto flujo en niños con bronquiolitis e insuficiencia respiratoria en piso de internación: Primera experiencia a nivel nacional. Arch Pediatría Urug. 2013;84:28-33.

34. URETA BL et al .Uso de VNI en una Unidad de cuidados intensivos cardiovasculares pediátricos. Factores de risco asociados al fracasso. Med. Lactente; 18 (1): 3-9, março 2011.

35. YUSTE ME, et al. Eficácia e segurança da oxigenoterapia com cânula nasal de alto fluxo na insuficiência respiratória hipercapnica moderada aguda. Revista Brasileira de Terapia Intensiva, Vol.31,no.2, São Paulo, ABR./juni, 2019. 